\title{
Midodrine: a role in the management of neurocardiogenic syncope
}

C R Ward, J C Gray, J J Gilroy, R A Kenny mended for the treatment of neurocardiogenic syncope in patients with frequent symptoms.

(Heart 1998;79:45-49)

Keywords: midodrine; neurocardiogenic syncope; head up tilt test

Setting-Cardiovascular investigation unit (a secondary and tertiary referral centre for the investigation and management of syncope).

Patients-16 outpatients (mean (SD) age 56 (18) years; five men) with frequent hypotensive symptoms (more than two syncopal episodes and fewer than 20 symptom free days per month), and reproducible syncope with glyceryl trinitrate (GTN) during head up tilt.

Design and intervention-Randomised double blind placebo controlled study. Patients were randomised to receive either placebo or midodrine for one month. Symptom events were recorded during each study month. At the end of each study month patients completed a quality of life scoring scale (Short Form 36) and a global assessment of therapeutic response. They received GTN with head up tilt for measurement of heart rate (electrocardiography), phasic blood pressure (digital photoplethysmography), and thoracic fluid index (transthoracic impedance plethysmography) during symptom provocation.

Results-Patients administered midodrine had an average of 7.3 more symptom free days than those who received placebo (95\% confidence interval (CI) 4.6 to 9; $\mathbf{p}<0.0001)$. Eleven patients reported a positive therapeutic response with midodrine $(p=0.002)$. All domains of quality of life showed improvement with midodrine, in particular physical function $(8.1 ; 95 \%$ CI 3.7 to 12.2 ), energy and vitality (14.6; 95\% CI 7.3 to 22.1 ), and change in health status (22.2; 95\% CI 11 to 33.4 ). Fourteen patients who were given placebo had tilt induced syncope compared with six given midodrine $(p=0.01)$. Baseline supine systolic blood pressure was higher and heart rate lower in patients who received midodrine than in those who were given placebo $(p<0.05)$. A lower thoracic fluid index in patients administered midodrine indicates increased venous return when supine and during head up tilt. There were no serious adverse effects.

Conclusions-Midodrine had a conspicuous beneficial effect on symptom frequency, symptoms during head up tilt, and quality of life. Midodrine is recom-
Syncope has many causes but the most common for all age groups is neurocardiogenic syncope, which is characterised by hypotension or bradycardia, or both, during symptoms. ${ }^{1}$ Upright posture in normal individuals increases venous pooling and is usually compensated for by a baroreflex mediated increase in heart rate and peripheral vasoconstriction. ${ }^{2}$ Vigorous contraction of an underfilled ventricle causes activation of afferent mechanoreceptors and in individuals susceptible to neurocardiogenic syncope this rapid increase in afferent neural activity is thought to produce a centrally mediated paradoxical decrease in heart rate and peripheral vasodilatation resulting in hypotension. ${ }^{34}$ The exact mechanism remains controversial, but reproduction of syncope during head up tilt is attributed to the described sequence of events often termed the Bezold Jarisch reflex.

A head up tilt table test consists of positioning the patient in a $70^{\circ}$ head up posture while monitoring heart rate and blood pressure. The test has been used increasingly to reproduce symptoms and haemodynamic changes in susceptible individuals with a clinical history suggestive of neurocardiogenic syncope..$^{5-8}$ Prolonged head up tilt is often used as a diagnostic test for neurocardiogenic syncope and its sensitivity is increased with concurrent administration of intravenous isoprenaline and sublingual or intravenous glyceryl trinitrate (GTN). ${ }^{9-11}$ Management of patients with recurrent neurocardiogenic syncope also remains controversial, in particular the role of pacing. ${ }^{12}$ Permanent pacing is appropriate for only a few patients with predominant cardioinhibition, while those with vasodepression are offered a range of treatments including $\beta$ blockade, disopyramide, theophylline, fludrocortisone, and etilefrine. There are few randomised placebo controlled studies of these medical treatments.

We have previously reported symptom relief in a man with recurrent vasodepressor syncope using midodrine, a specific $\alpha$ adrenergic receptor agonist with a low adverse effect profile. ${ }^{13}$ Midodrine binds to receptors of arteriolar and venous vasculature, thereby resulting in enhanced peripheral vascular tone and reduced venous pooling. 
The aim of this study was to assess whether midodrine influences symptom frequency and haemodynamic responses to head up tilt in patients with neurocardiogenic syncope.

\section{Patients and methods}

Patients in this double blind placebo controlled cross over study were randomised to receive either midodrine ( $5 \mathrm{mg}$ three times daily) or placebo for one month at the end of which there was a seven day wash out period during which no study medication was administered followed by a further month when alternative treatment was given. There were five study visits and medication was dispensed on the second and fourth visits. Patients were randomly assigned to the regimen using a computer program in the pharmacy department; the code was not broken until the end of the study. Each visit was scheduled at the same time of day and no food, sweetened drinks, or caffeine containing beverages were consumed before each visit. Patients were instructed to take one tablet three times daily: the first dose on arising in the morning, the second at midday, and the third during late afternoon but not after 1800 .

Thirty four patients with a history of frequent hypotensive symptoms of dizziness (more than two episodes per week), syncope (more than two episodes per month) attributed to neurocardiogenic syncope and who were not taking hypotensive medication and attended a syncope outpatient service were assessed. All patients underwent a passive head up tilt test to $70^{\circ}$ for 30 minutes (Akron foot plate assisted tilt table) with reproduction of hypotensive symptoms during head up tilt. Because a standard repeatable and comparative test was required in all patients, those with a positive passive head up tilt also underwent a head up tilt study to $70^{\circ}$ after administration of $400 \mu \mathrm{g}$ sublingual GTN (GTN-HUT). ${ }^{78}$ Symptomatic hypotension was defined as a reduction in systolic blood pressure of greater than $50 \mathrm{~mm} \mathrm{Hg}$ from baseline (resting for 15 minutes) with exact symptom reproduction during tilt. Blood pressure was continuously monitored during rest and tilt by phasic beat to beat measurement (digital photoplethysmography; Finapres: Ohmeda). ${ }^{14}$ Heart rate was recorded by surface electrocardiography and a third haemodynamic factor, the thoracic fluid index (TFI), was measured by transthoracic impedance plethysmography (Bomed: NCCOM3-R7; Kimal Products, Uxbridge, UK). ${ }^{16}$ Patients were initially rested supine for 15 minutes after which phasic blood pressure and heart rate were measured until steady baseline readings were obtained. $\mathrm{Pa}$ tients then received $400 \mu \mathrm{g}$ sublingual GTN and were tilted upright to $70^{\circ}$ with continuous measurement of haemodynamic variables for 30 minutes or until symptoms developed. The TFI measures thoracic resistance to the flow of a high frequency, low magnitude alternating current between electrodes placed at the root of the neck and the diaphragm. Conductivity of the thorax is determined by a delicate, but well defined balance among conductive blood and fluids, much less conductive intrathoracic tissues, and non-conductive air in the lungs. The TFI is therefore a non-invasive measure of subtle thoracic fluid change as seen in patients with decreased venous return during upright posture; less fluid in the thorax results in reduced conduction and thus larger TFI values.

Measurement of changes in patients daily symptoms and quality of life were coupled with the haemodynamic and symptomatic responses during GTN-HUT. Patients kept a daily record of hypotensive symptoms such as dizziness, falls, and blackouts. At the end of each study period assessment of the way in which hypotensive symptoms influenced daily living was aided by use of the Short Form 36 (SF 36), a standardised and semistructured quality of life assessment scoring scale. ${ }^{18}$ The SF 36 is a well validated tool that measures perceived health status by scoring responses to standardised questions in eight domains: (1) physical function; (2) role limitation: a) physical and b) mental; (3) social function; (4) mental health; (5) energy; (6) health perception; (7) change in health; and (8) pain. For the purpose of this study body pain scores were not completed. Patients also provided a global assessment of the therapeutic response to medication using a visual analogue scale: a positive response was taken as a score of more than 5 on a scale of 1 to 10 (poor to excellent).

GTN-HUT stimulus was repeated on the first visit to ensure a reproducible symptomatic and haemodynamic response; the patient was instructed to keep a diary card for one month. On review of the diary card at the second visit those with fewer than two syncopal events or more than 20 symptom free days or an asymptomatic GTN-HUT were excluded.

Sixteen patients were invited and agreed to participate in the study. All had reproducable hypotension and syncope during GTN-HUT on two separate study visits and symptom frequency as detailed earlier.

The initial dose of medication (midodrine/ placebo) for the first study period was then administered and one hour later blood pressure and heart rate were measured supine to ensure a systolic blood pressure value of no greater than $180 \mathrm{~mm} \mathrm{Hg}$ and a heart rate of no lower than 60 beats/min (peak serum concentrations of midodrine occur at one hour). Patients were discharged with instructions to take one tablet three times each day and record symptoms daily for one month.

On the third visit patients completed SF 36 and provided a global assessment of symptoms during the previous month. Patients then took a morning dose of medication and one hour later had a repeat GTN-HUT. Patients were asked to return after a seven day wash out period during which time no medication was taken.

On the fourth visit medication was supplied for the second treatment period and blood pressure and heart rate were recorded one hour after dosing. Patients were then discharged with a diary card. On the fifth visit patients completed SF 36 and provided global assessment of benefit from treatment. Patients had 
Table 1 Supine measurements and haemodynamic changes during GTN-HUT at the end of pretreatment (baseline), placebo, and midodrine study periods

\begin{tabular}{|c|c|c|c|c|c|c|}
\hline Study period & $\begin{array}{l}\text { Heart rate } \\
\text { (beats } / \mathrm{min} \text { ) }\end{array}$ & $\begin{array}{l}\text { Change in } \\
\text { heart rate }\end{array}$ & $S B P(m m H g)$ & Change in $S B P$ & $D B P(m m H g)$ & Time (mins) \\
\hline \multicolumn{7}{|c|}{ Steady state before tilt $(n=16)$} \\
\hline Baseline & $74.6(8)$ & & $127.0(18)$ & & $66.7(9)$ & \\
\hline Placebo & $75.5(9)$ & & $124.4(20)$ & & $66.2(11)$ & \\
\hline Midodrine & $69.8(9)^{\star}$ & & $141.4(26)^{\star}$ & & $70.4(13)$ & \\
\hline \multicolumn{7}{|c|}{ At the time of symptoms during tilt (positive responders only) } \\
\hline Baseline $(n=16)$ & $68(10)$ & $-7(11)$ & $66(25)$ & $-71(24)$ & & $6.4(2)$ \\
\hline Placebo $(\mathrm{n}=14)$ & $71(11)$ & $-9(8)$ & $65(22)$ & $-69(19)$ & & $6.3(3)$ \\
\hline Midodrine $(n=6)$ & $68(15)$ & $-6(12)$ & $71(16)$ & $-73(18)$ & & $8.8(2)$ \\
\hline
\end{tabular}

Values are mean (SD)

${ }^{\star} \mathrm{p}<0.05$.

SBP, systolic blood pressure; DBP, diastolic blood pressure.

GTN-HUT one hour after the morning dose of medication. All patients completed the study.

STATISTICAL ANALYSIS

The number of symptom free days, and supine blood pressure, heart rate and TFI values were normally distributed and a standard matched pairs $t$ test was used to test for treatment and carry over effects. Syncope during tilt and the therapeutic response are both binary outcomes and Prescott's test was used to test for treatment and carry over effects.

\section{Results}

Sixteen patients (five men; mean (SD) age 56 (18) years) were studied. All had symptomatic hypotension provoked by GTN-HUT at least twice and in the month before the study had a median of four syncopal events (range 2-8) and eight symptom free days (range $0-16$ ).

The study was a $2 \times 2$ cross over: group 1 received placebo for the first 28 days (period 1) and midodrine for the second (period 2); while group 2 received midodrine for period 1 and placebo for period 2. Six outcome variables were analysed: syncope during GTN-HUT; supine TFI; change in TFI during the first two minutes of tilt; the number of symptom free days; therapeutic response; and quality of life assessment (SF 36).

All patients had GTN-HUT at the end of the first and second study periods. There were four possible combinations of symptom responses during GTN-HUT: no syncope at the end of the first and second periods; syncope at the end of first and second periods; syncope at the end of the first period but not the second; or no syncope at the end of the first period but syncope at the end of the second. Eight patients showed the same response at the end of each period (two without syncope and six with syncope). The remaining eight, however, experienced syncope at the end of the placebo period but no syncope at the end of the midodrine period $(p=0.01)$. There was no significant carry over effect; therefore, midodrine significantly reduced reproduction of symptoms during provocative testing.

Heart rate and systolic blood pressure measured during stable state in a supine posture (mean of three readings) were significantly different between patients receiving placebo and those given midodrine. Patients who received midodrine had a lower heart rate and higher systolic blood pressure $(\mathrm{p}<0.01)$. Haemodynamic responses at the onset of symptoms during GTN-HUT in positive responders did not differ between patients given placebo and those administered midodrine; but the time taken to the onset of symptoms in patients who received midodrine was consistently prolonged than in those given placebo (table 1).

The supine TFI was similarly measured at the end of each study period. TFI was on average $1.48 \Omega$ lower in the midodrine period than in the placebo period ( $95 \%$ confidence interval (CI) 0.84 to $2.14 ; \mathrm{p}<0.01$ ) with no evidence of a treatment carry over effect. There were no patients with presyncopal symptoms or syncope during the initial two minutes of GTNHUT. The maximum increase in TFI at two minutes during GTN-HUT was on average $1.9 \Omega$ lower in patients given midodrine than those given placebo (95\% CI 1.1 to 2.7; $\mathrm{p}<0.01)$.

Fourteen patients experienced more symptom free days during the midodrine period; two patients experienced no symptom free days in either period (fig 1). Patients who received

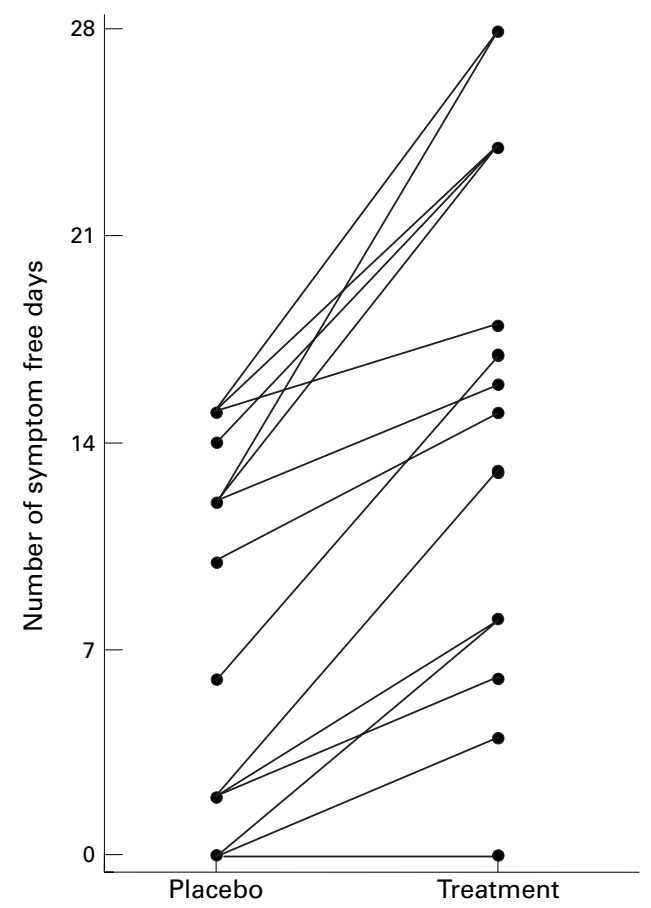

Figure 1 Number of symptom free days during midodrine (treatment) or placebo study periods. 
midodrine had an average of 7.3 more symptom free days than those given placebo ( $95 \%$ CI 4.6 to $9.9 ; \mathrm{p}<0.0001)$ with no carry over effect.

Patients were asked about their therapeutic response at the end of each treatment period. This was marked on a scale of 1 to 10 (poor to excellent) and dichotomised by taking a cut off greater than 5 as a positive response. This dichotomous response was analysed, with four possible combinations of responses: no therapeutic response in either the first or second periods; therapeutic response in the first and second periods; therapeutic response in the first period but not the second; and no therapeutic response in the first period but present in the second. No evidence of a carry over effect was found and so a formal test of treatment effect was performed. Five patients had no therapeutic response for either period, while the remaining 11 reported a positive therapeutic response after the midodrine period but not after placebo $(p=0.002)$.

Seven sets of quality of life scores from SF 36 were reviewed. Each set showed significantly improved scores during midodrine treatment. The results are expressed as the mean improvement in score between placebo and midodrine treatments, in particular physical function 8.1 (95\% CI 3.7 to 12.6 ), energy and vitality 14.6 (95\% CI 7.3 to 22.1 ), and change in health 22.2 (95\% CI 11.0 to 33.4). Four patients reported minor pilomotor reactions (goosebumps, tingling, and chills) with midodrine. Otherwise no adverse effects were reported.

\section{Discussion}

Neurocardiogenic syncope is characterised by a baroreceptor mediated hypotensive/bradycardic response to orthostatic stress. Head up tilt induces venous pooling with an associated decrease in cardiac output, which stimulates a baroreceptor mediated sympathetic response resulting in an increase in vascular tone and heart rate. ${ }^{34}$ This increased heart rate and inotropy coupled with reduced venous return, however, stimulates mechanoreceptors that may be situated in the wall of the left ventricle, which in susceptible individuals can trigger a centrally mediated withdrawal of sympathetic tone to the peripheral vasculature and an increased parasympathetic response, resulting in vasodilatation and bradycardia. The role of venous capacitance in enhancing venous pooling is unclear, but recent studies have indicated that calf vein volumes are increased in upright patients with syncope compared with those in asymptomatic controls. ${ }^{19}$

Recent concerns about the reproducibility of haemodynamic responses to head up tilt have questioned its role in assessing the efficacy of different therapeutic interventions. ${ }^{20}{ }^{21}$ Consequently, a placebo controlled cross over study design was used with GTN adminstration during head up tilt as a rigorous orthostatic stimulus.

Nitrates produce venous dilatation and hence a reduction in ventricular filling pressures and volumes that can cause circulatory collapse in susceptible individuals. ${ }^{22}$ Studies with sublingual and intravenous GTN have shown increased diagnostic yield for head up tilt while maintaining specificity for all adult age groups. ${ }^{78}$ Morillo et $a l^{23}$ and Fitzpatrick et $a l^{4}$ showed that $25 \%$ and $30 \%$, respectively, of patients had a negative third head up tilt result after two consecutive positive results. In this study patients were required to have two consecutive positive head up tilt test results with GTN before study entry, however only two of the 16 patients had a negative result with a third head up tilt test when given placebo. Patients with frequent and severe symptoms were chosen to participate in the study, thus justifying the short treatment period of one month. The wash out period ensured that symptom reproduction in the second treatment period was not influenced by syncope occurring during head up tilt at the end of the first treatment period

Management of patients with recurrent neurocardiogenic syncope remains controversial, in particular the role of pacing. Although the term vasovagal implies a significant bradycardic component in addition to hypotension, few people receive pacemakers because the dominant symptomatic response is due to hypotension. ${ }^{25} 26$ The criteria for pacing vary from no intervention to permanent physiological systems in patients who develop more than three second asystole or a bradycardia of less than 40 beats/min during orthostatic stress. ${ }^{27}$ Many pharmacological interventions have successfully prevented neurally mediated syncope by arterial vasoconstriction (etilefrine), suppression of the response to circulating catecholamines and sympathetic overactivity ( $\beta \quad 1$ adrenergic blocking agents, disopyramide, theophylline), or volume expansion (fludrocortisone), ${ }^{28-30}$ while other agents such as fluoxetine modify the baroreflex response centrally. To our knowledge there are only three published placebo controlled studies of pharmacological intervention ( $\beta$ blockers, disopyramide, and etilefrine) and they show no difference in the recurrence rate of syncope for patients treated with drug or placebo. ${ }^{23} 3031$ Midodrine is a potent peripherally acting $\alpha 1$ agonist that is well absorbed and rapidly metabolised to its active metabolite desglymidodrine in the systemic circulation. It has a plasma half life of approximately three hours. Oral or intravenous midodrine causes an increase in supine and standing blood pressure by increasing peripheral vascular resistance with a modest reduction in heart rate from counter regulatory enhancement of parasympathetic activity. This beneficial vasoconstrictor effect of midodrine compares favourably with that of other sympathomimetic agents ${ }^{32}{ }^{33}$ and the baseline supine haemodynamic data from this study support this effect of midodrine on systolic blood pressure and heart rate. Midodrine also decreases venous capacity, even at doses that do not substantially influence blood pressure: a single $5 \mathrm{mg}$ intravenous bolus of midodrine decreases venous capacity in healthy volunteers by up to $64 \%{ }^{34}$ The TFI, as measured by transthoracic impedance plethysmography, is a sensitive indicator of subtle changes in thoracic fluid content: the more fluid within the thoracic cavity the 
lower the TFI as resistance to the alternating current falls. The supine TFI and change in TFI during head up tilt were significantly lower during the midodrine period, supporting its role as a venoconstrictor. Midodrine may therefore have a role in treating neurocardiogenic syncope by promoting arterial vasoconstriction (to counter the falling blood pressure) and venoconstriction (to maintain preload), which coupled with its lack of $\beta$ agonist effects may prevent the onset of neurally mediated hypotension in susceptible patients. $^{35}$

The findings of this study support the role of midodrine in the treatment of neurocardiogenic syncope. Syncope rates during head up tilt were significantly reduced for a group of patients with recurrent syncope and reproducible vasodepressor response to head up tilt. These improved haemodynamic responses to head up tilt were coupled with significant reductions in hypotensive symptoms during the treatment period and unequivocal benefit in three major domains of quality of life: physical function, energy and vitality, and change in health. The impact of syncope on quality of life should not be underestimated. Linzer et $a l^{36}$ have shown that recurrent neurocardiogenic syncope can result in significant psychosocial and physical limitation similar to that experienced in patients with chronic rheumatoid arthritis or moderately severe chronic lung disease. ${ }^{36}$

The study group here is too small to predict which patients with neurocardiogenic syncope will most benefit from treatment with midodrine; however, the results support the use of midodrine in those with predominantly vasodepressor neurocardiogenic syncope.

1 Abi-Samra F, Malony JD, Fouad-Tarazi FM, et al. The usefulness of head-up tilt testing and haemodynamic investigation in the work up of syncope of unknown origin. $P A C E$ tion in the work

2 Hainsworth R, Al-Shamma YMH. Cardiovascular responses to upright tilting in healthy subjects. Clin $\mathrm{Sci}$ (Colch) 1988;74:17-22.

3 van Lieshout JJ, Wieling W, Karemaker JM, et al. The vasovagal response [editorial]. Clin Sci (Colch) 1991;81: $575-86$

4 Grubb BP, Kosinski D. Current trends in etiology, diagnosis, and management of neurocardiogenic syncope. Curr Opin Cardiol 1996;11:32-41

5 Hargreaves AD, Boon NA. Head-up tilt testing: the balance of evidence. Br Heart F 1994;72:216-17.

6 Kenny RA, Bayliss J, Sutton R. Head-up tilt: a useful test for investigating unexplained syncope. Lancet 1986;i:1352-4.

7 Benditt DG, Remole S, Balin S, et al. Tilt table testing for evaluation of neurally-mediated (cardioneurogenic) syncope: rationale and proposed protocols. PACE 1991;14: 1528-37.

8 Fitzpatrick A, Sutton R. Tilting towards a diagnosis in recurrent unexplained syncope. Lancet 1989;i:658-69.

9 Raviele A, Gasparini G, Menozzi C, et al. Value of head-up tilt testing potentiated with sublingual nitroglycerin to tilt testing potentiated with sublingual nitroglycerin to
assess the origin of unexplained syncope. Am $\mathcal{F}$ Cardiol assess the origin

10 McIntosh S, Lawson J, da Costa D, et al. Use of sublingual glyceryl trinitrate during head-up tilt: a provocative test for reproduction of neurocardiogenic symptoms in unexplained syncope. Cardiology in the Elderly 1996;4:33-7.

11 Almquist A, Goldenburg IF, Milstein S, et al. Provocation of bradycardia and hypotension by isoproterenol and uprigh posture in patients with unexplained syncope. $N$ Engl 7 Med 1989;320:346-51.

12 Grubb BP, Temesy-Amos P, Moore J, et al. Head-upright tilt table testing in evaluation and management of the maliganant vasovagal syndrome. Am f Cardiol 1992;69:904-8.

13 Ward CR, Kenny RA. Observations on midodrine in a case of vasodepressor neurogenic syncope. Clin Auton Res 1995; 5:257-60.

14 Petersen MEV, Williams TR, Sutton R. A comparison of non-invasive continuous finger blood pressure measurement (Finapres) with intra-arterial pressure during prolonged head-up tilt. Eur Heart f 1995;16:1647-54.

15 Imholz BPM, Settels JJ, van den Meiracker AH, et al. Noninvasive beat to beat finger blood pressure measurement during orthostatic stress compared to intraarterial presduring orthostatic stress compared

16 Kubicek WG, Karnegis JM, Patterson RP, et al. Development and evaluation of an impedance cardiac output ment and evaluation of an impedance car
system. Aerospace Medicine 1966;37:1208-12.

17 Kubicek WG, Karnegis JM, Patterson RP, et al. Impedance cardiography as a non-invasive method to monitor cardiac function and other parameters of the cardiovascular system. Ann NY Acad Sci 1970;170:724-32.

18 McHorney CA, Ware JE, Raczek AE. The MOS 36ItemShort Form Health Survey (SF-36) II. Psychometric and clinic al tests of validity in measuring physical and mental health constructs. Med Care 1993;31:247-63.

19 Hargreaves A, Muir A. Lack of variation in venous tone potentiates vasovagal syncope. Br Heart f 1992;67:486-90.

20 Moya A, Permanyer-Miralda G, Sagrista-Sauleda J, et al. Limitations of head-up tilt test for evaluating the efficacy of therapeutic interventions in patients with vasovagal syncope: results of a controlled study of etilefrine versus placebo. F Am Coll Cardiol 1995;25:65-9.

21 Kapoor WN, Smith MA, Miller NL. Upright tilt testing in evaluating syncope: a comprehensive literature review. $\mathrm{Am}$ F Med 1994;97:78-88.

22 Come PC, Pitt B. Nitroglycerin-induced severe hypotension and bradycardia in patients with acute myocardial infarction. Circulation 1976;54:624-8.

23 Morillo CA, Leitch JW, Yee R, et al. A placebo controlled trial of intravenous and oral disopyramide for prevention of neurally mediated syncope induced by head-up tilt. $7 \mathrm{Am}$ Coll Cardiol 1993;22:1843-8.

24 Fitzpatrick A, Ahmed R, Williams S, et al. A randomised trial of medical therapy in "malignant vasovagal syndrome" or "neurally-mediated bradycardia/hypotension syndrome". European fournal of Cardiac Pacing and Electrophysiology 1991;2:99-102.

25 Goldstein DS, Spannarkel M, Pitterman A, et al. Circulatory control mechanisms in vasodepressor syncope. Am Heart F 1982;104:1071-5.

26 Sra JS, Anderson AJ, Sheikh SH, et al. Unexplained syncope evaluated by electrophysiological studies and head-up tilt testing. Ann Intern Med 1991;114:1013-19.

27 Sutton R, Peterson M, Brignole M, et al. Proposed classification for tilt-induced vasovagal syncope. European fournal of Cardiac Pacing and Electrophysiology 1992;2:1803.

28 Milstein S, Buetitikofer J, Dunnigan A, et al. Usefulness of disopyramide for prevention of upright tilt induced hypotension-bradycardia. Am f Cardiol 1990;65:1339-44.

29 Ferguson DW, Thomes MD, Mark AL. Effects of propranolol on reflex vascular responses to orthostatic stress in
humans: role of baroreceptors. Circulation 1983;67:802-7.

30 Natale A, Sra J, Dhala A, et al. Efficacy of different treatment strategies for neurocardiogenic syncope. PACE 1995;18: 655-62.

31 Brignole M, Menozzi C, Gianfranchi L, et al. A controlled trail of acute and long term medical therapy in tilt-induced neurally mediated syncope. Am f Cardiol 1992;70:339-42.

32 Jankovic J, Hiner BC, Brown DC. Neurogenic orthostatic hypotension: a double-blind, placebo-controlled study with midodrine. Am f Med 1993;95:38-48.

33 Kaufmann H, Brannan T, Krakoff L, et al. Treatment of orthostatic hypotension due to autonomic failure with a peripheral alpha-adrenergic agonist (midodrine). Neurology 1988;38:951-6.

34 Erhringer $\mathrm{H}$. Studies on the peripheral haemodynamics in man after intravenous infusion of a small dose of di-1-(2',5'-dimethoxyphenyl)-2-glycinamido-ethanol-(1)hydrochloride. Int $\mathcal{f}$ Clin Pharmacol Ther Toxicol 1971;4: 415-20.

35 Piwinski SE, Jankovic J, McElligot MA. A comparison of post space flight orthostatic intolerance to vasovagal syncope and autonomic failure and the potential use of the alpha-agonist for these conditions. F Clin Pharmacol 1994; 34:466-71.

36 Linzer M, Pontinen M, Gold D. Impairment of physical and psychosocial function in recurrent syncope. f Clin Epidemiol 1991;44:1037-43. 